\title{
Trends in RF Simulation Algorithms
}

\author{
Joel R. Phillips \\ Cadence Berkelev Laboratories, Berkelev, CA 94704 \\ Dan Feng \\ Cadence Dosign Systems, San Jose, CA 95134
}

\begin{abstract}
Continued growth in the market for wireless communication devices has resulted in renewed interest in algorithms for computer-aided design of RF systems. In this article we review some recent progress in the field of circuit-level $\mathrm{RF}^{2}$ simulation algorithms. Comparisons of the relative advantages of popular simulation approaches are presented, with particular attention to the relation between numerical simulation strategics and the nature of RF circuit problems.
\end{abstract}

\section{INTRODUCTION}

Roughly speaking, the primary task of the analog portion of a wireless system is to shift data signals in frequency. A frequency shift of an analog data stream can be accomplished, ideally, by multiplication with an auxiliary periodically time-varying signal. Thus a central characeristic of RF circuits is that they are usually driven with one or more periodic signals. These periodic signals establish a set of frequency ranges about which the data signals cluster in a narrow band. This makes the RF circuit simulation problem difficult, because the response of a circuit over both fast (because of the high frequency carrier signals) and slow (because of the small frequency spacing of the other signals) timescales must be determined. The RF simulation problem is tractable because, though signals must be resolved with very fine frequency spacing, only a small portion of the overall frequency band is involved in the circuit operation. Specialized algorithms for RF simulation have been around for some time. This paper is concerned with recent developments and experience with new numerical techniques. The reader is directed to the book (Kundert 
et al., 1990) and the reviews (Kundert, 1999; Gilmore and Steer, 1991) for further background and bibliographic material.

\section{RF SIMULATION AND THE PERIODIC STEADY-STATE}

The simplest non-trivial example of a circuit with a sparse signal spectrum is a circuit that develops a periodic steady-state response to a periodic drive signal. The methods used in solving periodic steady-state problems form the basis of the more complicated analysis methods such as linear time-varying noise analysis and distortion analysis via quasi-periodic steady-state methods.

Formulating steady-state problems. The system of $n$ differential-algebraic equations that describes the RF circuit can be written in the general form

$$
\frac{d}{d t} q(v(t))+i(v(t))=u(t)
$$

where $t \in \Re$ is the time variable, $v(t) \in \Re^{n}$ is the circuit state (such as a collection of node voltages), $q, i: \Re^{n} \rightarrow \Re^{n}$ represent dynamic and static components in the circuit, and $u(t) \in \Re^{n}$ represents the effect of external sources. The periodic steady-state problem involves, given an excitation $u(t)$ such that $u(t+T)=u(t)$ for some fundamental period $T$, finding a solution to Eq. 1.1 such that $v(t+T)=v(t)$ is periodic.

The simplest way to find a steady-state solution is to simply integrate Eq. (1.1) forward in time from a known initial condition until the solution $v(t)$ becomes periodic. Such an approach discards any potential efficiency that could be gained from the sparsity of the signal spectrum, and is impractical since it may take a very long time for the circuit to reach steady-state. Instead what is done is to discretize and solve Equation (1.1) over one period, subject to the periodic boundary condition $v(t+T)=v(t)$.

Discretization. Two methods are presently popular. Finite difference discretizations approximate the state variables $v(t)$ with piecewise-polynomials in $t$. For example, by using a first-order accurate backward difference approximation to the time-derivative, the backward-Euler integration rule

$$
\frac{q(v(t+h))-q(v(t))}{h}+i(v(t+h))=u(t+h)
$$

may be used to solve Equation (1.1). If an equation similar to Equation (1.2) is written at $m$ timepoints in an interval $\left[t_{0}, t_{0}+T\right]$ and the periodic boundary condition applied as $v\left(t_{0}\right)=v(t+T)=v\left(t_{m}\right)$, then a nonlinear system of equations in the $m n$ variables $v\left(t_{k}\right), k=1 \ldots m$ is obtained. Each timepoint will be coupled to $p+1$ others if an order- $p$ polynomial is used to discretize 
the derivative in Equation (1.1). When $v(t), q(v(t))$, or $i(v(t))$ contain rapid transitions, as happens in highly nonlinear circuits, the local timestep and order of the method can be locally varied to accurately resolve the unusual behavior without an efficiency penalty.

Spectral methods, such as used in harmonic balance, represent the second popular class of discretizations. Spectral discretizations use a high-order, usually global, representation of the functions $v(t), i(v(t)), q(v(t))$. A typical choice is to expand, say, the unknown state variables $v(t)$ in a series of complex exponentials (or, equivalently, sines and cosines), as

$$
v(t)=\sum_{k=-K}^{K} c_{k} e^{i k \omega_{0} t}
$$

where $\omega_{0}=2 \pi / T$ is the fundamental frequency. Note that the periodic boundary condition is automatically satisfied.

If the representation of the function in terms of the frequency-domain coefficients $c_{k}$ is known, then the a time-derivative can be computed by multiplying the $k$ th Fourier coefficient by $i k i \omega_{0}$. If enough harmonics are chosen to adequately represent $v(t)$, then spectral differentiation is essentially exact. For smooth signals, the approximation error decreases rapidly as the number of terms $K$ in the expansion increases and then spectral methods can achieve high accuracy at low cost. On the other hand, if the voltages or currents, or their low-order derivatives, have sharp or irregular features, whether as a result of circuit operation or due to device models, the spectral representation may converge quite slowly, resulting in an inefficient algorithm.

In modern codes, the nonlinear functions are evaluated in the time-domain, but the time-differentiation operation takes place in the frequency domain, and therefore an efficient means of translating a solution quickly between the domains is needed. The translation operation usually uses the fast Fourier transform (FFT), and this usually imposes constraints on the timestep spacing that create difficulties when dealing with signals containing rapid transitions. Recently, efforts have been made to reformulate the spectral methods to use non-uniform timestep spacing(Nastov and White, 1999).

Harmonic balance methods can naturally include linear distributed elements in simulations. After Fourier transformation the convolution $y(t)=$ $\int_{-\infty}^{t} h .(t-\tau) x(\tau) d \tau$ that describes the distributed element becomes the simple multiplication, $Y(\omega)=H(\omega) X(\omega)$ where $Y(\omega), H(\omega), X(\omega)$ are the frequency-domain versions of $y, h$, and $x$ respectively. Finite-difference based methods must either evaluate the convolution directly in the time domain, an expensive operation, or translate the solution to the frequency domain. It is difficult to achieve efficient time/frequency conversions for signals with nonequispaced timepoints, and so finite-difference based methods are not well suited to analyzing circuits with many distributed elements. 
(a)
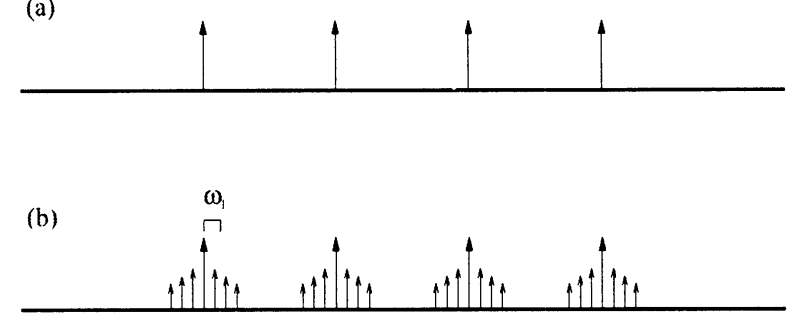

(c)

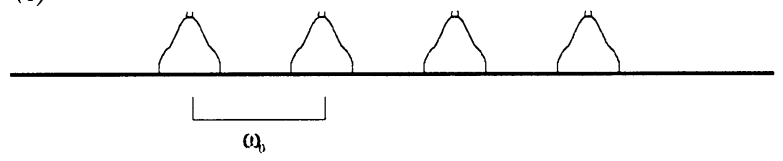

Fïgure 1 Periodic (a), quasi-periodic (b), and broadband (c) RF data signals.

\section{MULTI-FREQUENCY ANALYSES}

Most practical problems have signals at multiple frequency scales. Figure (1) shows three common RF data signal profiles. If the frequencies of all the signals are harmonically related, as in Figure 1 (a), then the problem can be solved using periodic steady-state methods, with the fundamental periodic the least common multiple of the periods of the various excitations. However, often signals are present at many unrelated frequencies. If the circuit response can be described by frequencies that are harmonics of several distinct fundamental frequencies, as in Figure 1 (b), the circuit has a quasi-periodic steady state that can be solved for directly. For non-quasi-periodic signals that have a spectrum that is dense in a narrow band around a set of (quasi-)periodically spaced frequencies, but unstructured, as in Figure 1 (c), "envelope" techniques can be used. There are various ways of formulating the "multiple-frequency" analyses.

PDE methods. A signal is quasi-periodic if it possesses a Fourier-series expansion in multiple frequencies, such as

$$
v(t)=\sum_{k} \sum_{l} c_{k, l} e^{i k \omega_{0} t} e^{i l \omega_{1} t}
$$

for a two-frequency signal with fundamentals $w_{0}, w_{1}$. An obvious way to solve the quasi-periodic problem is by a spectral method : truncating the series (1.4) and solving for the coefficients $c_{k l}$. When the signals around the carrier 
frequency have non-periodic character, a representation such as

$$
v(t)=\sum_{k=-K_{0}}^{k=k_{0}} c_{k}(t) e^{i k \omega_{0} t} .
$$

may be assumed . A broader class of algorithms can be obtained by making the observation (Brachtendorf et al., 1996; Roychowdhury, 1997) that Equations (1.4) and (1.5) have some of the flavor of a two-dimensional Fourier series expansion.

Suppose we define the two-variable quantity

$$
V\left(t_{1}, t_{2}\right)=\sum_{k=-K_{0}}^{k=K_{0}} \sum_{l=-K_{1}}^{K_{1}} c_{k, l} e^{i k \omega_{0} t_{1}} e^{i l \omega_{1} t_{2}} .
$$

Note that if $V\left(t_{1}, t_{2}\right)$ is known, then $v(t)$ can be recovered from the "diagonal" as $v(t)=V(t, t)$. If we likewise define multi-dimensional inputs $U\left(t_{1}, t_{2}\right)$, and replace the time-derivative in Eq.(1.1) by the two-dimensional gradient operator, then, neglecting for the moment distributed terms, the DAE (1.1) becomes a quasi-linear hyperbolic partial differential-algebraic equation

$$
\left[\frac{d}{d t_{1}}+\frac{d}{d t_{2}}\right] q\left(V\left(t_{1}, t_{2}\right)\right)+i\left(V\left(t_{1}, t_{2}\right)\right)=U\left(t_{1}, t_{2}\right)
$$

It can be shown that if $V\left(t_{1}, t_{2}\right)$ is a solution of the "multivariate partial differential equation" (MPDE), (1.7), then $V(t, t)$, which lies on the "characteristics" of the PDE, is a solution to the DAE (1.1). The original circuit simulation problem can thus be recast as the problem of solving a particular partial differential equation. It should be clear that if periodic boundary conditions are imposed on both variables of the MPDE, and a spectral discretization used in both dimensions, then a representation equivalent to the multi-tone harmonic balance formulation is obtained.

The emergence of the MPDE viewpoint is potentially interesting for three reasons. First, it provides a more formal framework for analyzing methods for solving the "multiple timescale RF simulation problem." Second, it suggests potentially new algorithms. Virtually any scheme that can be used to solve a hyperbolic PDE can be used in the RF simulation context. For example, discretizing the PDE with finite-difference methods along both dimensions produces "time-time" (Roychowdhury, 1997) approaches that are not obviously obtained in other contexts. Third, practical experience with algorithms for solving RF problems could potentially be applied to develop algorithms for solving hyperbolic partial differential equations with multiple-timescale solutions.

Because the PDE formulations embed the one-dimensional quantity $v(t)$ into a higher dimensional space, there is no guarantee of efficiency, and in 
fact unless the signals in the circuit satisfy certain conditions, the methods can be less efficient than traditional transient simulation. Generally if the signal bandwidth is large relative to the carrier frequency, there is no advantage to the PDE methods. For envelope-like solutions, Equation (1.5), the equivalent statement is that the $c_{k}(t)$ must vary slowly on a timescale of the order of $1 / \omega_{0}$ for the methods to be both efficient and accurate.

Sampling Methods. The idea behind sampling methods is to remove the difficulties caused by the high-frequency carrier by sampling all the signals in the circuit at multiples of the carrier period $T_{0}=1 / \omega_{0}$. To see how this might work, consider sampling the signal $v(t)=\sum_{k=-K_{0}}^{k=K_{0}} c_{k}(t) e^{i k \omega_{0} t}$ at a discrete set of points $t_{n}^{\prime}=t_{0}+n T_{o}$ where $t_{0} \in\left[0, T_{o}\right)$ and $n$ runs over the integers. The result is a signal $\bar{v}\left(t_{n}^{\prime}\right)=\sum_{k=-K_{0}}^{k=K_{0}} c_{k}\left(t_{n}^{\prime}\right) e^{i i_{0} t_{0}}$ defined at the discrete time instants $t_{n}^{\prime}$. Note that the fast time variation due to the high carrier frequency has disappeared. If the signals are concentrated in frequency around the carrier harmonics, then the $c_{k}\left(t_{n}^{\prime}\right)$ are slowly varying.

In the quasi-periodic case, where each $c_{k}\left(t_{n}^{\prime}\right)$ can be represented by a few terms in a Fourier series, $c_{k}\left(t_{n}^{\prime}\right)=\sum_{l} c_{k l} l^{l \omega_{1} t_{n}^{\prime}}$ the $c_{k l}$ at consecutive timepoints, $t_{n}$ and $t_{n+1}=t_{n}+T_{0}$ and must be related by the quasi-periodic boundary condition $c_{k l}\left(t_{n+1}^{\prime}\right)=c_{k l}\left(t_{n}^{\prime}\right) e^{i \omega_{1} T_{0}}$. This boundary condition must relate the signals at the timepoints $t_{n}^{\prime}$ and $t_{n+1}^{\prime}$ that are the beginning and ending points of a single carrier cycle. For envelope-like solutions, boundary conditions are obtained by requiring that the $c_{k}(t)$ lie on a low-order polynomial. Because of the use of special boundary conditions of this sort, only a few cycles actually need be simulated, and the cost is relatively independent of the relation between the various frequency scales.

When applied to multi-frequency problems, the sampling algorithms can also be thought of as ways to solve the special PDE (1.7). However, they differ from the normal PDE-based implementations in that the solution is actually performed directly on the "characteristics" of the hyperbolic PDE and generally the different cycles on the characteristic are coupled together at a single, common point in the "clock" phase. The sampling methods thus always compute solutions to the DAE (1.1). Error can only be induced as a result of the boundary conditions. Note that, unlike the PDE methods, the sampling schemes are intrinsically one-dimensional. In cases where the PDE methods might become inefficent, the sampling schemes reduce to conventional timedomain simulation algorithms.

\section{NONLINEAR SOLUTION TECHNIQUES}

Once the circuit equations have been formulated and discretized, a highdimensional nonlinear system of equations must be solved. The basic Newton 
method to solve the nonlinear system $f(x)=0$ is the iterative application of the rule $x^{k+1}=x^{k}-J\left(x^{k}\right)^{-1} f\left(x^{k}\right)$ for $k=1,2, \ldots$ until converged, where $J\left(x^{k}\right)$ is the Jacobian matrix of of $f\left(x^{k}\right), J_{i j}\left(x^{k}\right)=\partial f_{i} / \partial x^{j}$. For a discretization of an $n$-equation DAE with $m$ timepoints, the Jacobian will be a rank- $m n$ matrix. As an example, consider the simple backward-Euler discretization of the periodic-steady-state problem, which has the Jacobian

$$
J=\left[\begin{array}{cccc}
\frac{C_{1}}{h_{1}}+G_{1} & & -\frac{C_{M}}{h_{1}} \\
-\frac{C_{1}}{h_{2}} & \frac{C_{2}}{h_{2}}+G_{2} & \\
& \ddots & \ddots & \\
& & -\frac{C_{M-1}}{h_{M}} & \frac{C_{M}}{h_{M}}+G_{M}
\end{array}\right]
$$

where

$$
C_{k}=\left.\frac{\partial q(v)}{\partial v}\right|_{v\left(t_{k}\right)}, \quad G_{k}=\left.\frac{\partial i(v)}{\partial v}\right|_{v\left(t_{k}\right)} .
$$

For certain types of finite difference schemes, alternative strategies to the direct Newton updates are available that can prove advantageous. Consider for the moment the periodic-steady-state problem, and suppose Equation 1.1 is discretized using a "causal" low-order difference rule. That is, the rule is such that if an initial condition $v\left(t_{0}\right)$ is known, the rule can be used to propagate the solution to Equation (1.1) forward in time, one timestep at a time. Now imagine that one point on the periodic solution happens to be known. All of the other points on the periodic solution can be easily obtained simply by integrating the DAE (1.1) forward in time.

To make the argument concrete, define the transition function $\phi\left(v_{0}, t_{k}, t_{f}\right)=$ $v\left(t_{f}\right): v(t)$ satisfies equation (1.1) for $t \in\left[t_{k}, t_{f}\right]$ and $v\left(t_{k}\right)=v_{0}$. In terms of the transition function, the periodic boundary condition is expressed as $v_{0}=\phi\left(v_{0}, t_{0}, t_{0}+T\right)$. The shooting method consists of solving the periodicsteady-state problem by solving this alternative equation. To do so we must solve a linear system involving the shooting method Jacobian which is $I-\Phi$ where the matrix $\Phi$ is the sensitivity of the transition function to the initial condition,

$$
\Phi=\frac{\partial \phi\left(v_{0}, t_{0}, t_{0}+T\right)}{\partial v_{0}}
$$

Consider again Equation (1.8). If we let $L$ denote the lower block-triangular portion of this matrix, and $U$ the upper-block-triangular portion, then $L^{-1} J=$ $I+L^{-1} U$. It turns out that the last size $n \times n$ block in the matrix $L^{-1} U$ is the matrix $\Phi$. $\Phi$ can be computed from knowledge of $J$, and conversely systems involving $J$ can be easily solved if the matrix $\Phi$ can be inverted. 
The difference between using shooting methods to solve the finite-difference equations and the "normal" Newton iteration is in the way the finite-difference equations are updated after every Newton iteration. In the direct approach, an update to the entire waveform over the period $T$ is made simultaneously. In the shooting method, at first only one point, the first point in the interval, is updated. Then a sequence of local nonlinear problems are solved to be sure that the DAE (1.1) is satisfied at every point in the interval $\left[t_{0}, t_{0}+T\right)$. When the end of the interval is reached, the value of the transition function for the new Newton iteration has been computed. If the solution is not yet periodic, another Newton update to the initial condition $v_{0}=v\left(t_{0}\right)$ can now take place. Empirically it is observed that the shooting method has better global convergence properties than direct updates of the finite-difference equations. By acting on the function $\phi$ instead of the individual difference equations, highly nonlinear behavior ocurring in the interior of the periodic interval may be hidden from the Newton iteration.

The shooting method can be naturally extended to solve the equations arising from the sampling schemes, since those schemes depend only on finding the values of the states $v$ at a single point in each of $K$ cycles. The boundary condition that relates the beginning and endpoints of the cycle are expressed using a $K \times K$ matrix $D_{K}$, resulting in the the nonlinear system $\left(D_{K} \otimes\right.$ $\left.I_{n}\right) v_{K}-\phi_{K}\left(v_{K}\right)=0$, where $\otimes$ is the Kronecker product, $I_{n}$ is the $n$ by $n$ identity matrix, and $\phi_{K}$ represents is a multi-cycle transition function that represents the transition functions of the $K$ sample points. The dimension $K n \times K n$ Jacobian of the Newton iteration will be $J_{K}=D_{K} \otimes I_{n}-\Phi_{K}\left(v_{K}\right)$ where $\Phi_{K}$ is a block-diagonal matrix whose blocks are the Jacobians of the individual sensitivity functions. Shooting methods may also be used to solve the equations resulting from finite-difference discretizations of MPDE equation formulations.

For difficult RF simulation problems, continuation methods(Allgower and Georg, 1990) are the procedure of choice to achieve global convergence of the Newton iteration. Continuation methods achieve convergence of the Newton iteration by solving a sequence of nonlinear problems, each of which is easy to solve because the solution of the previous problem provides a starting value for the Newton iteration that is close to the actual solutuion. For example, when performing intermodulation distortion analysis with one of the sampling methods described previously, the Newton iteration may have difficulty converging at high power levels, and so the power is gradually increased from a low level where the solution is known, until the desired operating point is reached (Feng et al., 1999). 


\section{SMALL SIGNAL ANALYSIS}

In some cases, for example, in noise analysis, there are inputs to the RF circuit whose amplitudes are small and who generate small amplitude responses. Performing a linear-time varying analysis of the circuit can be computationally advantageous when there are many such inputs at frequencies unrelated to the larger carrier or RF data signals. The first step in the linear time-varying analysis is to linearize the RF circuit around a time-varying operating point that is set by the large signals in the circuit, for example the local oscillator signal in a mixer. The linearization procedure can be accomplished by separating the excitation and responses in Equation (1.1) into large and small-signal parts and then taking the first term in the Taylor series expansion of the response about the large signal. The resulting time-varying linear system is $\frac{d}{d t}\left[C\left(v_{s}(t)\right) v_{s}(t)\right]+G(v(t)) v_{s}(t)=$ $u_{s}(t)$ where $u_{s}(t)$ describes the small-signal inputs and $v_{s}(t)$ is the small-signal response, and $v(t)$ is the large-signal bias point.

Conversion Matrices. The defining characteristic of linear time-varying operators is that they shift frequency. A small-signal input at a frequency $\omega$ will induce a circuit response in a periodically time-varying circuit at frequencies that are offset by multiples of the fundamental frequency. Thus signals at frequencies $w+k \omega_{0}$, with $k$ an integer, are mutually coupled by the timevariation. We can represent these relations by defining an infinite-dimensional "vector" of the inputs and responses at the various harmonic offsets. $\tilde{v}_{s}(w)=$

$$
\left[v_{s}^{T}\left(\omega-K \omega_{0}\right) \cdots v_{s}^{T}(\omega) \cdots v_{s}^{T}\left(\omega+K \omega_{0}\right)\right]^{T} \cdot \tilde{u}_{s}(w)-\left[u_{s}^{T}\left(\omega-K \omega_{0}\right) \cdots u_{s}^{T}(\omega) \cdots\right]^{T}
$$

that are related by the conversion matrices (Maas, 1988) by $\overline{v_{s}}(\omega)=\tilde{H}(\omega) \tilde{u}_{s}(\omega)$ where

$$
\tilde{H}=\left[\begin{array}{cccccc}
\ddots & & & & \\
& H_{-1,-1} & H_{-1,0} & H_{-1,1} & \\
\cdots & H_{0,-1} & H_{0,0} & H_{0,1} & \cdots \\
& H_{1,-1} & H_{1,0} & H_{1,1} & \\
& & & & \ddots
\end{array}\right]
$$

and each sub-matrix $H_{k, l}$ represents a transfer function matrix that converts signals from freqency $\omega+l \omega_{0}$ to frequency $\omega+k \omega_{0}$.

Given an operating point and a linearization, i.e. a Jacobian matrix such as (1.8), computing the conversion matrices is conceptually straightforward. For example, it has been shown in (M. Okumura et al., 1993; Telichevesky et al., 1996) that the conversion matrices associated with finite-difference discretizations are given by (apart from a diagonal scaling) the inverse of the matrix $L+\alpha(\omega) U$ where $L$ and $U$ respectively are again the lower and upper blocktriangular pieces of the Jacobian (1.8) and $\alpha$ is a frequency-dependent scalar constant. 
Noise Analysis. One of the most important applications of the time-varying small-signal analysis is to compute noise in RF circuits. As the noise signals are stochastic process, we are usually concerned with computing quantities such as power spectral densities or noise correlation matrices. If $L$ is a linear operator and $x$ an element in its domain, then it it a general property of linear systems(Papoulis, 1991) that $E\{L x\}=L E\{x\}$ and likewise for the adjoint operator $L^{*}$. Often we have a system with input $x$, output $y$, and we wish to calculated a correlation quantity $S_{y}, S_{y}=E\left\{y y^{*}\right\}$. This is easy to do, as $E\left\{y y^{*}\right\}=E\left\{L x(L x)^{*}\right\}=E\left\{L x x^{*} L^{*}\right\}=L E\left\{x x^{*}\right\} L^{*}$, and so $S_{y}=L S_{x} L^{*}$. For example, if we let $\tilde{x}$ and $\tilde{y}$ represent the input and output respectively of an RF circuit with conversion matrix $\tilde{H}(\omega)$, then the matrices of (cyclostationary) power spectral densities, $S_{x}(\omega)$ and $S_{y}(\omega)$ are related by (Roychowdhury et al., 1998) $S_{y}(\omega)=\tilde{H}(\omega) S_{x}(\omega) \tilde{H}(\omega)^{H}$ where superscript $H$ denotes Hermitian transpose. A similar relation can be derived to relate time-domain representations of noise.

Oscillators and phase noise. The accurate estimation of noise in oscillators is of particular interest to RF designers and requires particular care. In (Kaertner, 1990) a microscopic theory that describes calculation of the stationary part of the phase noise spectrum was presented. Recently, a simple phenomenological time-varying theory has appeared (Hajimiri and Lee, 1998), and a more detailed statistical treatment of the microscopic phase noise analysis problem has been performed (Demir et al., 1998). The time-varying linear noise analysis in Section 5. can be used for phase noise calculations, however, care must be taken when interpreting the results, as the linearized analysis is best interpreted as a specific computational procedure used to perform calculations in the context of the more rigorous theories, rather than an independent theory of phase noise.

\section{KRYLOV-SUBSPACE SOLVERS}

The major computational obstable in RF simulation is to quickly solve the equations that come from linearizations of the discretized circuit DAEs. One innovation that has enabled the simulation of very large circuits is the use of modern linear algebra techniques, in particular, Krylov-subspace based linear system solution algorithms. These algorithms require only the computation of a matrix-vector product in order to solve a linear system. In both the finitedifference and spectral formulations (Telichevesky et al., 1995; Telichevesky et al., 1996; Melville et al., 1995), the matrix-vector products needed by the Krylov-subspace solvers can be computed in time and memory that is nearly linear in the size of the circuit. However, the performance of Krylov-subspace algorithms can be very problem-dependent, and so their effective use requires close integration of linear algebra techniques with a knowledge of the specific 
simulation problem and choice of the numerical formulation. This is done by means of a technique called preconditioning.

Convergence of the Krylov-subspace algorithms for solving the matrix equation $A x=b$ is roughly determined by the location of the eigenvalues of $A$. In RF analysis, the matrix $A$ is a matrix whose blocks are generated from the small circuit Jacobian matrices $C$ and $G$. Practical circuits usually have widelyvarying physical time constants, and so the matrices assembled from $C$ and $G$ tend to be intrinsically ill-conditioned. Furthermore, forming the large matrices that occur in direct solution of the equations of spectral and finite-difference discretizations tends to exacerbate any underlying ill-conditioning.

Fortunately, the matrices resulting from most RF equation formula have a structure that can be exploited in constructing a preconditioner. A typical approach (Melville et al., 1995; Long et al., 1997) is to approximate the timevarying $C$ and $G$ matrices by constant or piecewise-constant approximations. The resulting preconditioner is block-sparse (block-diagonal in the simplest case) with sparse blocks, and so can be easily inverted. Particularly effective preconditioners can be constructed for the sampling formulations (Feng et al., 1999). In this case, it is the frequency-averaged transition function Jacobian that is used to construct a block-diagonal preconditioner. These preconditioners are not as sensitive to nonlinear behavior as their harmonic-balance counterparts because the dominant nonlinear behavior, the response of the circuit to the carrier signal, is hidden by the transition function.

Optimizations to the Krylov-subspace algorithms can often be made for small-signal problems. The key idea is that the Krylov space of a matrix $\alpha I+A$ is invariant with respect to the complex number $\alpha$. This means that if a linear system is solved with one $\alpha$, a second linear system can re-use the matrix-vector products that were computed during the first solve thereby accelerating the iterative solution process (Telichevesky et al., 1996). In principle, all the formulation and discretizations lead to small-signal analyses where block or solvers can be applied, however, they are particularly useful for the shooting-based solution of small-signal equations based on finite-difference discretization of a periodic-steady-state operating point. Essentially the shooting method converts the continuous time problem to a discrete time problem, with system matrix $z I-A, z$ being the complex frequency.

\section{SUMMARY}

Advanced numerical techniques have greatly advanced the state-of-the-art in RF circuit simulation over the past few years. Progress in detailed simulation will continue to derive from deeper understanding of the relation between the generic numerical methods described in Sections 4. and 6. with the RF simulation formulations presented in Sections 3. and 5. 


\section{ACKNOWLEDGMENTS}

The authors would like to acknowledge Ken Kundert, Jess Chen, Baolin Yang, Alper Demir, Jaijeet Roychowdhury and Jacob White for useful discussions that have contributed to the preparation of this article.

\section{REFERENCES}

Allgower, A. and Georg, K. (1990). Numerical Continuation Methods. SpringerVerlag, New York.

Brachtendorf, H. G., Welsch, G., Laur, R., and Bunse-Gerstner, A. (1996). Electrical Engineering, 79:103-112.

Demir, A., Mehrotra, S., and Roychowdhury, J. (1998). In Proc. 35th Design Automation Conference, pages 26-31, San Francisco, CA.

Feng, D., Phillips, J., Nabors, K., Kundert, K., and White, J. (1999). In Proc. 36th Design Automation Conference, pages 635-640, New Orleans, LA.

Gilmore, R. and Steer, M. (1991). Int. J. Microwave and Millimeter Wave Computer Aided Engineering, 1(1):159-180.

Hajimiri, A. and Lee, T. (1998). IEEE J. Sol. State Circuits, 33:179-193.

Kaertner, F. X. (1990). Int. J. Circuit Theory and Applications, 18:485-519.

Kundert, K. (1999). IEEE J. Sol. State Circuits, to appear September 1999.

Kundert, K. S., White, J. K., and Sangiovanni-Vincentelli, A. (1990). SteadyState Methods for Simulating Analog And Microwave Circuits. Kluwer Academic Publishers, Boston.

Long, D., Melville, R., Ashby, K., and Horton, B. (1997). In Proc. Custom Integrated Circuits Conference.

M. Okumura, H. T., Itakura, T., and Sugawara, T. (1993). IEEE Trans. Circuits and Systems - I, 40:581-590.

Maas, S. (1988). Nonlinear microwave circuits. Artech House, Norwood, MA. Melville, R., Feldmann, P., and Roychowdhury, J. (1995). In Proc. Custom Integrated Circuits Conference.

Nastov, O. and White, J. (1999). In Proc. 36th Design Automation Conference, pages 641-646, New Orleans, LA.

Papoulis, A. (1991). Probability, random variables, and stochastic processes. McGraw Hill, New York.

Roychowdhury, J. (1997). In Proc. 34th Design Automation Conference, pages 269-274, Anaheim, CA.

Roychowdhury, J., Long. D., and Feldmann, P. (1998). IEEE J. Sol. St. Circuits, 33:324-336.

Telichevesky, R., White, J., and Kundert, K. (1995). In Proc. 32rd Design Automation Conference.

Telichevesky, R., White, J., and Kundert, K. (1996). In Proc. 33rd Design Automation Conference. 